\title{
The Effect of the Field of Adaptation on Chromatic Discrimination Ellipses
}

\author{
Ágnes Urbin ${ }^{1 *}$, Balázs Vince Nagy¹, Klára Wenzel ${ }^{1}$ \\ 1 Department of Mechatronics, Optics and Mechanical Engineering Informatics, Faculty of Mechanical Engineering, Budapest \\ University of Technology and Economics, Múegyetem rkp. 3, 1111 Budapest, Hungary \\ *Corresponding author, e-mail: urbin@mogi.bme.hu
}

Received: 17 November 2021, Accepted: 13 January 2022, Published online: 17 February 2022

\begin{abstract}
In this paper results of chromatic discrimination measurements obtained in two lighting scenarios are compared. Chromatic discrimination thresholds were measured in relation with systematically manipulated chromaticities seen under different visual angles. In both cases the Ellipse module of the Cambridge Colour Test (CCT) was obtained binocularly by normal colour-observers. Discrimination ellipses fitted to the measured thresholds were compared in terms of the adapting chromaticity and the visual angle of the adapting stimulus.

Our findings show that changes in the reference chromaticity of the CCT have stronger effect on the elongation of the chromatic discrimination ellipses compared to the effect of the adapting chromaticity obtained in a light booth.

Further comparison of discrimination thresholds estimated towards the confusion directions and the corresponding radii of the Ellipse test results suggests that the reliability of the Ellipse test results depends on the relation between the measurement directions and the confusion directions.
\end{abstract}

Keywords

chromatic adaptation, visual field, discrimination threshold, discrimination ellipse, Cambridge Colour Test (CCT)

\section{Introduction}

Chromatic adaptation is a process which provides approximately constant appearance of colours under lights with different spectral content. With the definition of the International Lighting Vocabulary (ILV) by CIE, chromatic adaptation is the "visual process whereby approximate compensation is made for changes in the colours of stimuli, especially in the case of changes in illuminants" [1].

Over the years, chromatic adaptation transforms (CATs) based on different theories were developed and embedded into colour appearance models (CAMs) to predict the adapting shift and the degree of adaptation caused by the chromatic changes of the environment $[2,3]$.

The baseline of chromatic adaptation is the perception of white. As the ILV defines, incomplete adaptation is the "phenomenon in which the adopted white in a given viewing environment does not actually appear white to an observer" [1].

Even though we experience in everyday life that chromatic adaptation performs quite well under white lights, the increasing number of applications of LED-based light sources might raise the question of limitations and extensions of the currently accepted adaptation models under coloured lights or incomplete adaptation [4-10].

The variability of parameters of the visual field such as the distribution of luminance and colours indicates that besides the generic aim of creating comprehensive models, studies aiming to model scenarios defined with a selected range of parameters, such as colours of self-luminous stimuli, or unrelated colours can be found in the literature [11-14].

The main metrics of CAMs are the corresponding colours: two colour stimuli having the same colour appearance under two sets of chromatic adaptation conditions [2]. Nevertheless, chromatic discrimination is also affected by chromatic adaptation [15-19].

Since the fundamental colour matching measurement of MacAdam [20], chromatic discrimination ellipses estimating the areas in chromaticity diagrams, which cannot be discriminated by the observers, are applied in colour science to describe colour diagrams with Riemannian metrics and to analyse the perceptual uniformity of colour spaces [21-23]. 
Therefore, measuring chromatic discrimination thresholds under different states of chromatic adaptation shall provide information about the changes in the perceived colour space. The goal of our study was to observe the effect of adapting chromaticities seen under different visual angles on chromatic discrimination ellipses of normal colour-observers.

\section{Methods}

In our study chromatic discrimination measurements in two lighting scenarios were obtained and compared. In both cases the Ellipse module of the Cambridge Colour Test (CCT) was obtained binocularly by normal colour-observers.

Cambridge Colour Test is a computer-based pseudoisochromatic test [24], in which the task is to find the orientation of a Landolt-C figure. In the Ellipse module chromatic discrimination thresholds are measured from a reference chromaticity towards a selected number of equally spaced directions in the CIE 1976 UCS diagram.

Besides the thresholds, an ellipse fitted with leastsquares method to the measured vectors is calculated. In our cases 8 measurement directions were applied and the reference chromaticity varied. Test images were shown until response, but for 3 seconds at the longest.

Input and output chromaticity coordinates $\left(u^{\prime} ; v^{\prime}\right)$, the measured discrimination thresholds $\left(\Delta E_{u^{\prime} v^{\prime}}\right)$ and further calculated parameters are interpreted in the CIE 1976 UCS diagram.

\subsection{Stimulus}

The pseudoisochromatic figures were displayed on a MultiSync ${ }^{\circledR}$ FP2141SB ${ }^{\text {TM }}$ Display with 20 inches diagonal image size $(1600 \times 1200$ pixels $)$, controlled with ViSaGe MkII, after gamma correction performed with a ColorCAL MkII colorimeter. The diameter of the pseudoisochromatic figures was 11.7 inches. The viewing distance was 3 meters providing that the gap of each Landolt-C figure was shown under $1^{\circ}$ visual angle.

\subsection{Visual fields}

The main difference between the two scenarios is the structure of the visual field.

In each case chromatic discrimination thresholds were measured in relation with systematically manipulated chromaticities. The above-mentioned chromatic stimulus appeared in different areas of the visual field.

\subsubsection{Unaided eyes - case A}

First, measurements were obtained in a darkened room with unaided eyes. The adapted white was considered as equal energy white, defined by the darkened room. The reference chromaticities of the discrimination measurements were the chromaticities of the backgrounds of the pseudoisochromatic figures, which varied in the experiment as the independent variable (see Fig. 1 (a)).

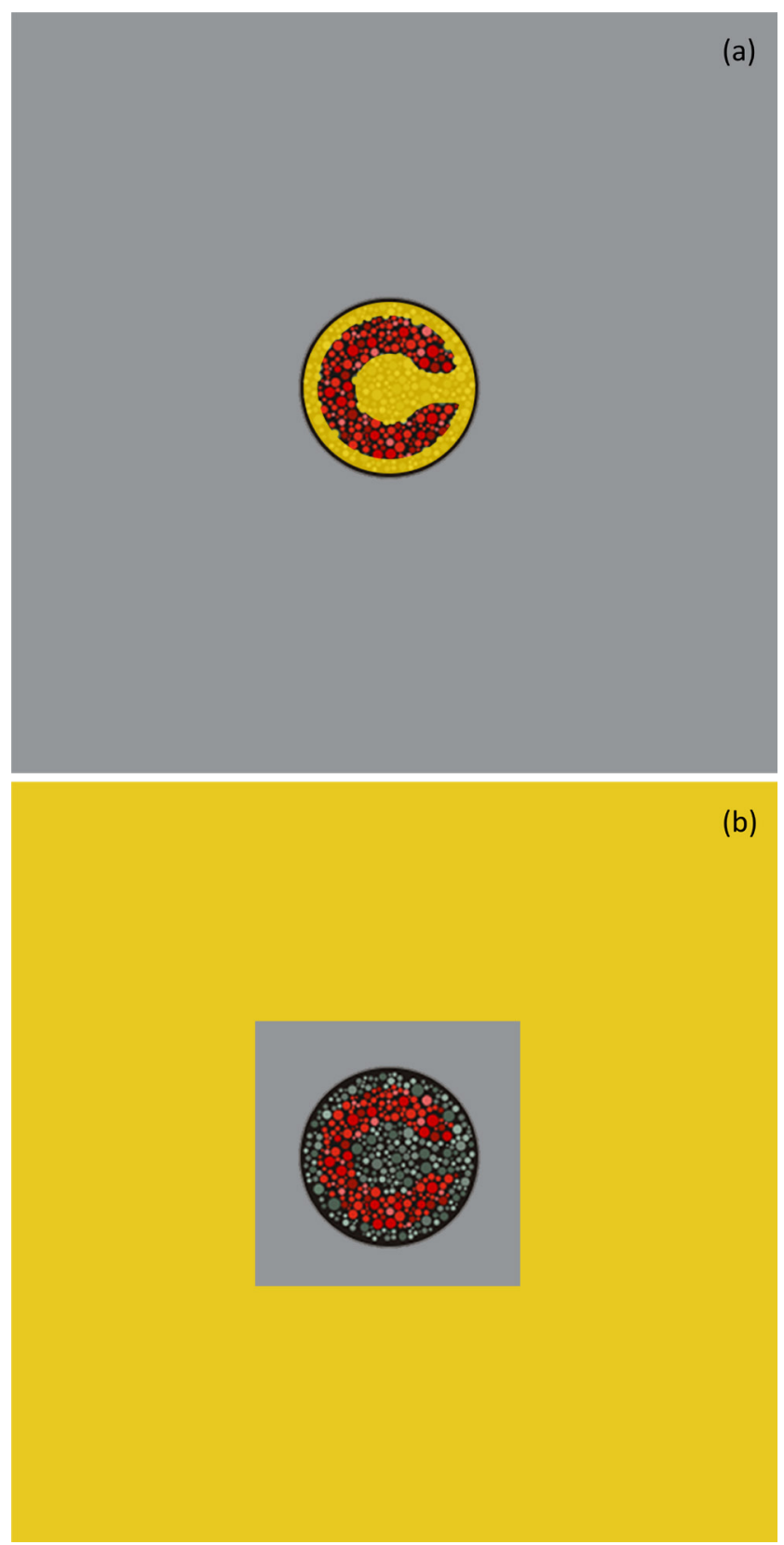

Fig. 1 Visual fields of the experiments described in the paper obtained with unaided eyes (a) and in the light booth (b). Yellow colour indicates the areas where the systematically manipulated chromaticity appeared. 


\subsubsection{Light booth - case B}

Second, subjects were sitting in a custom made spectrally tuneable light booth during measurements. The test figures were displayed behind the light booth, seen over a window within $8^{\circ}$ of visual angle. The ambient lighting in the booth covered most of the visual field, except the window at the back of the booth and the test display behind (see Fig. 1 (b)).

In the light booth five primaries of high intensity LEDs were controlled (red - $623 \mathrm{~nm}$, amber - $596 \mathrm{~nm}$, green $527 \mathrm{~nm}$, blue - $460 \mathrm{~nm}$ and warm white) providing full field homogeneous stimulus of luminance level set to $26 \mathrm{~cd} / \mathrm{m}^{2}$ for all illumination conditions.

Reference chromaticity of the ellipse tests was fixed to a white point, while the chromaticity of the light on the booth varied as the independent variable.

\subsection{Experimental design, procedure and subjects}

In both cases measurements were performed binocularly in a dark room after five minutes provided for dark adaptation, while the instructions were explained.

The test procedure was explained to the subjects showing the first test image of the test sequence, with a colour difference of 0.0011 in terms of $\Delta E_{u^{\prime} v^{\prime}}$, between the background and the target. Subjects had to detect the orientation of the Landolt-C test figure randomly selected of 4 options and record the answer with a remote controller.

In both experiments normal colour vision was a condition of participation. Subjects were university students. Normal colour vision was validated based on Trivector test results: thresholds measured towards the Protan, Deutan and Tritan confusion points. Normal colour vision was assumed if the thresholds did not exceed $100 \times 10^{-4}$ towards either the Protan or the Deutan confusion point or $150 \times 10^{-4}$ towards the Tritan confusion point [24] in terms of $\Delta E_{u^{\prime} v^{\prime}}$.

\subsubsection{Unaided eyes - case A}

In case $\mathrm{A}$ the neutral point was assumed to equal energy white $(0.2024 ; 0.4689)$. Reference points were defined along eight directions equally spaced and centred on the neutral point (see Fig. 2). The reference points follow the experimental design of a previously published experiment of the authors [25].

In the experiment 4 subjects (21 to 37 years old) participated, each of them obtained the measurements five times in reference points along two perpendicular reference directions. Ellipses were fitted to the average threshold values in each reference point.

Luminance levels were set to $5 \pm 3 \mathrm{~cd} / \mathrm{m}^{2}$.

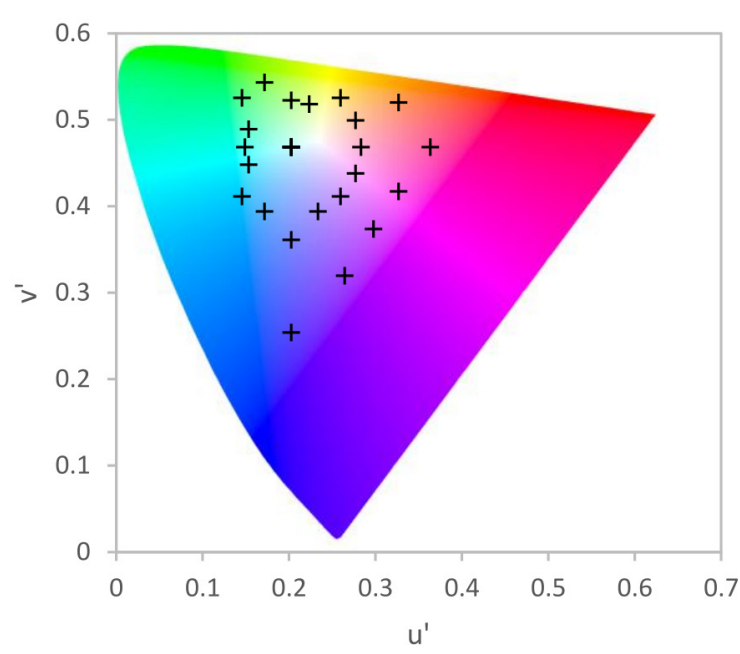

Fig. 2 The 23 reference points positioned along the eight reference directions centred on the neutral point - case A

Obtaining one set of measurements took approximately 30 minutes, including dark adaptation, prior instructions, trial tests, the main test sequence, and short breaks.

\subsubsection{Light booth - case $B$}

In case $B$ the reference chromaticity was fixed to $(0.1977 ; 0.4689)$ - the default setting of the CCT. The chromaticity of the ambient light in the booth varied as shown in Fig. 3.

In the experiment 18 subjects (15 to 38 years old) participated. The luminance of the ambient light in the booth was $26 \mathrm{~cd} / \mathrm{m}^{2}$ and the luminance levels of the CCT were set to the default setting of the CCT: $13 \pm 5 \mathrm{~cd} / \mathrm{m}^{2}$.

\section{Experimental results and analysis}

In analysis ellipses fitted to the average thresholds with least-squares method were applied. Since the luminance values of the display and the ambient light were different

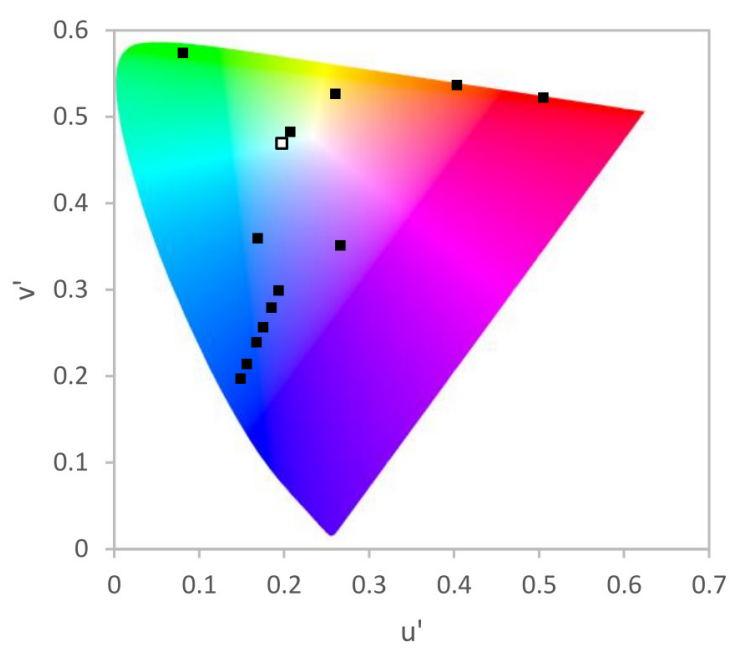

Fig. 3 The adapting chromaticities in the light booth - case B 
in the two cases, the areas of the ellipses were not directly comparable. Therefore, the orientations and the axis-ratios of the ellipses were analysed. In Fig. 4 and Fig. 5 the radii of the ellipses are multiplied by 3 .

\subsection{Ellipses}

The ellipses fitted to the average threshold values measured with unaided eyes - case A, are shown in Fig. 4. The centres of the ellipses represent the reference chromaticity coordinates of the measurements.

Fig. 5 shows the ellipses fitted to the average threshold values measured in the light booth - case B. The centres of

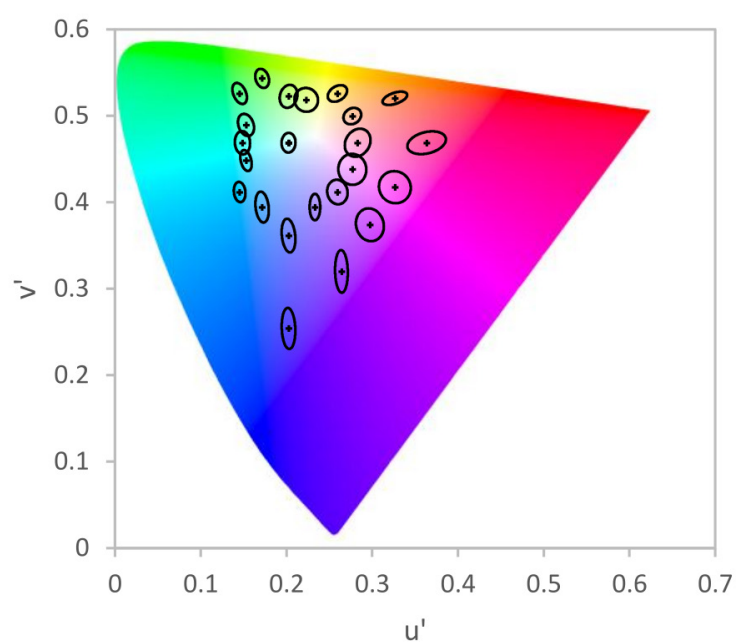

Fig. 4 Ellipses fitted to the average chromatic discrimination thresholds measured with unaided eyes. The centres of the ellipses represent the reference chromaticity of the CCT test figures. The radii of the ellipses are multiplied by 3 .

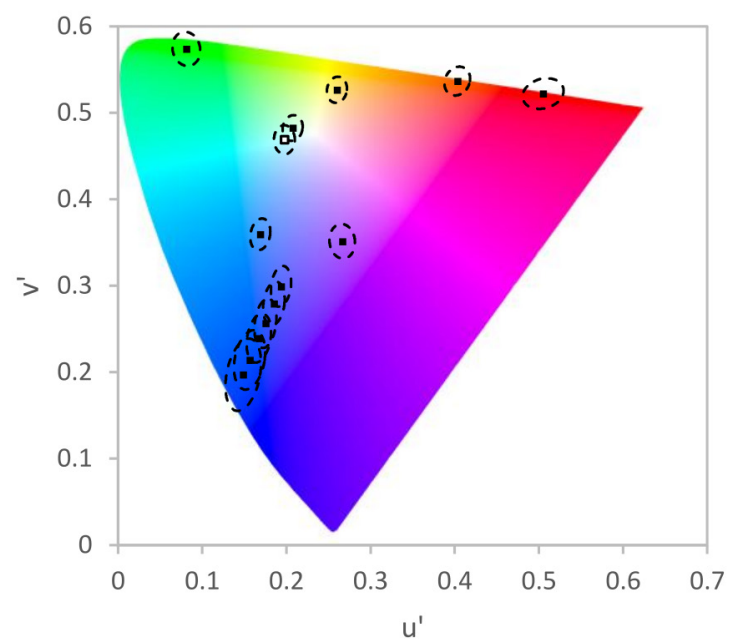

Fig. 5 Ellipses fitted to the average chromatic discrimination thresholds measured in the light booth. The centres of the ellipses represent the chromaticity of the light booth, while the reference chromaticities of the CCT test figures were $(0.1977 ; 0.4689)$ in each case. The radii of the ellipses are multiplied by 3 . the ellipses represent the chromaticity coordinates of the ambient lighting of the light booth during measurements.

The neutral point $(0.1977 ; 0.4689)$, which was the reference point of all CCT measurements in this case, is denoted with the white square.

\subsection{Reference directions vs. ellipse axes}

In the analysis $\delta$ denotes the angle between the $u^{\prime}$ abscissa and the segment of the neutral point and the ellipse centre (further on reference direction), and $\theta$ denotes the angle between the $u^{\prime}$ abscissa and the major axis of the ellipses (further on ellipse orientation).

Fig. 6 shows the scatter plot of the reference directions $(\delta)$ towards the ellipse orientations $(\theta)$ in each adapting chromaticity.

The scatter plot and correlation analysis show a strong, positive, linear relationship between $\delta$ and $\theta$ in both cases. Spearman's correlation coefficients and $p$-values are in case A: corr. coeff. $=0.8194 ; p=0.7281 \mathrm{E}-06$ and in case $\mathrm{B}$ : corr. coeff. $=0.9560 ; p=3.3650 \mathrm{E}-07$.

These correlations describe that the ellipses are elongated towards the adapting chromaticities in both cases.

\subsection{Excitation purity vs. axis ratio}

Even though the directions of the elongations correlate with the adapting chromaticities in both cases A and B, Fig. 4 and Fig. 5 indicate that the axis ratios of the ellipses are affected in different rates.

To observe this difference, ellipses of cases A and B were grouped based on reference directions. Six reference

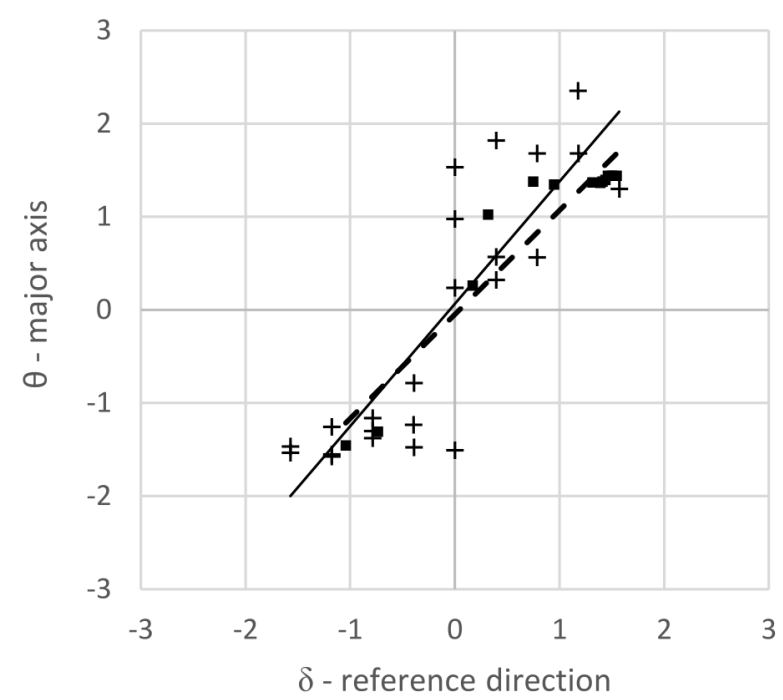

Fig. 6 Reference directions vs. major axis orientations. Crosses and solid trendline denote data of case A, squares and dashed trendline denote data of case B. 
directions were selected, along with ellipses of both cases A and B were defined. Ellipses measured along the same reference directions were compared.

Since the CIE 1976 UCS is not a perfectly uniform colour space and the adapting chromaticities are distributed across the chromaticity diagram in diverse directions, evaluation in terms of $\Delta E_{u^{\prime} v^{\prime}}$ colour differences may generate an error of non-uniformity of the colour diagram.

To avoid this error, excitation purity of the adapting chromaticities were calculated in the 1976 UCS diagram and the axis ratios of the ellipses were compared accordingly, as shown in Fig. 7.

In Fig. 7 axis ratio of the ellipses is shown in the function of the excitation purity of the reference chromaticity. Markers with crosses (+) denote data from measurements with unaided eyes - case A, and markers with solid contour ( $\square$ ) denote data from measurements obtained in the light booth - case B. The background colours of the markers denote the reference directions along with the reference points were defined. Corresponding pairs of markers are linked with solid line. Fig. 7 shows that the axis ratio of ellipses measured with unaided eyes $(+)$ is higher than that of ellipses measured in the light booth ( $\square$ ) towards each of the six observed reference directions.

\subsection{Thresholds towards the confusion lines}

In case A chromatic discrimination thresholds towards the three confusion lines were estimated following the authors' former publication [25], further on referred as Trivector estimates. The radii of the Ellipse test results towards the

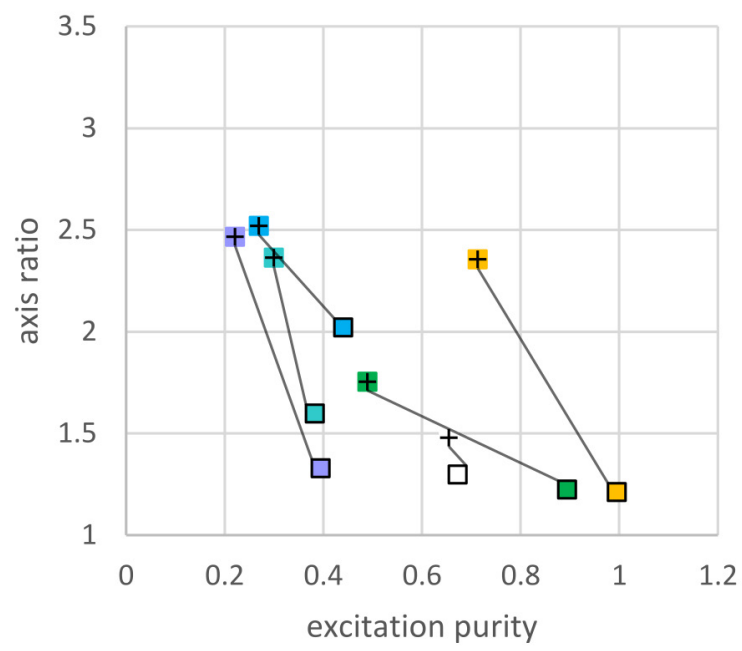

Fig. 7 Excitation purities (calculated in the 1976 UCS diagram) vs. axis ratios. Crosses denote data of case A, squares denote data of case B.

Linked data points belong to the same reference direction. confusion directions were also calculated based on Eq. (1), further on referred as Ellipse estimates:

$$
r(\vartheta)=\sqrt{\frac{a^{2} \times b^{2}}{a^{2} \times \sin ^{2}(\vartheta)+b^{2} \times \cos ^{2}(\vartheta)}}
$$

where:

$r$ denotes the dependent variable - the radius,

$\vartheta$ denotes the angle to the radius from the major axis, $a$ denotes the radius on the major axis, and

$b$ denotes the radius on the minor axis.

The ratios of the Trivector and the Ellipse estimates towards the three confusion lines are shown in Fig. 8.

As Fig. 8 shows, Trivector estimations exceeded the Ellipse estimations in almost all cases.

\section{Conclusion}

In our study chromatic discrimination ellipses of normal colour observers measured under two sets of chromatic adaptation conditions were compared.

We aimed to investigate the effect of the location and area of the adapting chromaticity in the visual field.

In the case of measurements obtained with unaided eyes the surround was dark and the adapting chromaticity appeared as the reference chromaticity of the CCT test figure. In the other case reference chromaticity of the CCT was grey and measurements were obtained in a light booth providing different coloured surround.

Our findings show that the adapting chromaticities affected the orientation of the ellipses in a similar way: the ellipses elongated towards the adapting chromaticity.

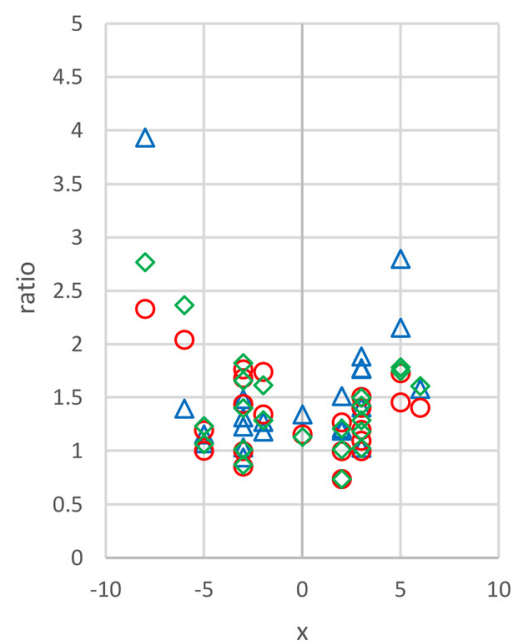

Fig. 8 The ratios of the Trivector and Ellipse JND estimates towards the Protan (red circles), Deutan (green diamonds) and Tritan (blue triangles) in terms of reference distance $(x)$. 
The elongation of the ellipses was affected in a different rate. Axis ratios of the ellipses were observed in terms of excitation purity. Changes in the reference chromaticity of the CCT test figures have stronger effect on the elongation of the chromatic discrimination ellipses compared to the effect of the adapting chromaticity obtained in the light booth.

The Trivector estimates exceeded the threshold values calculated from the Ellipse measurements at the same reference point in almost all cases, indicating that there are colors outside the estimated chromatic discrimination ellipses, which the subjects cannot discriminate.

\section{References}

[1] International Commission on Illumination "CIE S 017/E:2020 ILV: International Lighting Vocabulary, 2nd Edition", CIE Central Bureau, Vienna, Austria, 2020. https://doi.org/10.25039/S017.2020

[2] Fairchild, M. D. "Color Appearance Models", John Wiley \& Sons Ltd, Chichester, UK, 2013. https://doi.org/10.1002/9781118653128

[3] Fairchild, M. D. "Von Kries 2020: Evolution of degree of chromatic adaptation", Color and Imaging Conference, 2020(28), pp. 252-257, 2020.

https://doi.org/10.2352/issn.2169-2629.2020.28.40

[4] Peng, R., Luo, M. R., Cao, M. "Modelling incomplete chromatic adaptation on a display under different ambient illuminations", Color and Imaging Conference, 2019, pp. 231-236, 2019. https://doi.org/10.2352/issn.2169-2629.2019.27.41

[5] Lee, S.-H., Lee, M.-H., Sohng, K.-I. "Factors of Incomplete Adaptation for Color Reproduction Considering Subjective White Point Shift for Varying Illuminant", IEICE Transactions on Fundamentals of Electronics, Communications and Computer Sciences, E91.A(6), pp. 1438-1442, 2008. https://doi.org/10.1093/ietfec/e91-a.6.1438

[6] Fairchild, M. D. "Formulation and testing of an incomplete-chromatic-adaptation model", Color Research and Application, 16(4), pp. 243-250, 1991.

https://doi.org/10.1002/col.5080160406

[7] Huang, H.-P., Wei, M., Ou, L.-C. "White appearance of a tablet display under different ambient lighting conditions", Optics Express, 26(4), pp. 5018-5030, 2018.

https://doi.org/10.1364/oe.26.005018

[8] Smet, K. A. G., Zhai, Q., Luo, M. R., Hanselaer, P. "Study of chromatic adaptation using memory color matches, Part I: neutral illuminants", Optics Express, 25(7), pp. 7732-7748, 2017. https://doi.org/10.1364/OE.25.007732

[9] Smet, K. A. G., Zhai, Q., Luo, M. R., Hanselaer, P. "Study of chromatic adaptation using memory color matches, Part II: colored illuminants", Optics Express, 25(7), pp. 8350-8365, 2017. https://doi.org/10.1364/oe.25.008350

[10] Ma, S., Hanselaer, P., Teunissen, K., Smet, K. A. G. "Effect of adapting field size on chromatic adaptation", Optics Express, 28(12), pp. 17266-17285, 2020.

https://doi.org/10.1364/OE.392844
This suggests that the reliability of the Ellipse test depends on whether one(s) of the measuring directions equally splitting $360^{\circ}$ coincide or approach one of the confusion directions. To overcome this, the measurement of chromatic discrimination thresholds towards the Protan, Deutan and Tritan confusion points is recommended, even in the case of the examination of subjects with normal color vision.

\section{Acknowledgement}

Authors thank all subjects who participated in the measurements for their contribution.

[11] Li, C., Li, Z., Wang, Z., Xu, Y., Luo, M. R., Cui, G., Melgosa, M., Brill, M. H., Pointer, M. "Comprehensive color solutions: CAM16, CAT16, and CAM16-UCS", Color Research and Application, 42(6), pp. 703-718, 2017. https://doi.org/10.1002/col.22131

[12] Hermans, S., Smet, K. A. G., Hanselaer, P. "Color appearance model for self-luminous stimuli", Journal of the Optical Society of America A, 35(12), pp. 2000-2009, 2018. https://doi.org/10.1364/josaa.35.002000

[13] Huang, W. J., Yang, Y., Luo, M. R. "Verification of the CAM15u colour appearance model and the QUGR glare model", Lighting Research \& Technology, 51(1), pp. 24-36, 2019. https://doi.org/10.1177/1477153517734402

[14] Gao, C., Li, C., Shi, K., Luo, M. R., Pointer, M. R. "CAM20u:An extension of CAM16 for predicting the color appearance of unrelated colors", Color Research and Applications, 46(4), pp. 749-758, 2021. https://doi.org/10.1002/col.22615

[15] Loomis, J. M., Berger, T. "Effects of chromatic adaptation on color discrimination and color appearance", Vision Research, 19(8), pp. 891-901, 1979. https://doi.org/10.1016/0042-6989(79)90023-3

[16] Krauskopf, J., Gegenfurtner, K. "Color discrimination and adaptation", Vision Research, 32(11), pp. 2165-2175, 1992. https://doi.org/10.1016/0042-6989(92)90077-V

[17] MacAdam, D. L. "Influence of chromatic adaptation on color discrimination and color perception", Die Farbe, 4, pp. 133-143, 1955.

[18] Webster, M. A., Wilson, J. A. "Interactions between chromatic adaptation and contrast adaptation in color appearance", Vision Research, 40(28), pp. 3801-3816, 2000. https://doi.org/10.1016/S0042-6989(00)00238-8

[19] Cohen, J. D. "Temporal independence of the Bezold-Brücke hue shift", Vision Research, 15(3), pp. 341-351, 1975. https://doi.org/10.1016/0042-6989(75)90081-4

[20] MacAdam, D. L. "Visual Sensitivities to Color Differences in Daylight", Journal of the Optical Society of America, 32(5), pp. 247-274, 1942. https://doi.org/10.1364/JOSA.32.000247

[21] Xu, Q., Zhao, B., Cui, G., Luo, M. R. "Testing uniform colour spaces using colour differences of a wide colour gamut", Optics Express, 29(5), pp. 7778-7793, 2021. https://doi.org/10.1364/oe.413985 
[22] Pant, D. R., Farup, I. "Riemannian formulation and comparison of color difference formulas", Color Research and Applications, 37(6), pp. 429-440, 2012.

https://doi.org/10.1002/col.20710

[23] Chao, J., Lenz, R., Matsumoto, D., Nakamura, T. "Riemann Geometry for Color Characterization and Mapping", Conference on Colour in Graphics, Imaging and Vision, 2008, pp. 277-282, 2008.
[24] Mollon, J. D., Regan, B. C. "Cambridge Colour Test: Handbook", Cambridge Research Systems Ltd., Rochester, UK, 2000.

[25] Urbin, Á., Nagy, B. V. "Chromatic Discrimination Thresholds as a Function of Color Differences and Cone Excitations", Periodica Polytechnica Mechanical Engineering, 65(4), pp. 385-397, 2021. https://doi.org/10.3311/ppme.18691 